\title{
Two-dimensional Ising model with self-dual biaxially correlated disorder
}

\author{
Farkas Á. Bagaméry, ${ }^{1,2}$ Loïc Turban, ${ }^{2, \text { 母 }}$ and Ferenc Iglói ${ }^{3,1,0}$ \\ ${ }^{1}$ Institute of Theoretical Physics, Szeged University, H-6720 Szeged, Hungary \\ ${ }^{2}$ Laboratoire de Physique des Matériaux, Université Henri Poincaré (Nancy 1), BP 239, \\ F-54506 Vandouvre lès Nancy Cedex, France \\ ${ }^{3}$ Research Institute for Solid State Physics and Optics, H-1525 Budapest, P.O.Box 49, Hungary
}

(Dated: November 21, 2018)

\begin{abstract}
We consider the Ising model on the square lattice with biaxially correlated random ferromagnetic couplings, the critical point of which is fixed by self-duality. The disorder, which has a correlator $G(r) \sim r^{-1}$, represents a relevant perturbation according to the extended Harris criterion. Critical properties of the system are studied by large scale Monte Carlo simulations. The correlation length critical exponent $\nu=2.005(5)$ corresponds to that expected in a system with isotropic correlated long-range disorder, whereas the scaling dimension of the magnetization density $x_{m}=\beta / \nu=$ $0.1294(7)$ is somewhat larger than in the pure system. Conformal properties of the magnetization and energy density profiles are also examined numerically.
\end{abstract}

\section{INTRODUCTION}

The presence of quenched disorder of different types is an inevitable feature of real materials. The effect of randomness on the physical properties of systems is often dramatic, in particular in the vicinity of singular points, such as for classical phase transitions at finite temperature or for quantum phase transitions at $T=0$. In a random system the set of critical exponents associated with a phase transition can be completely different from that in the nonrandom system, which means that disorder can be a relevant perturbation in the sense of the Harris criterion. 1 In most of the studies of disordered systems the parameters (couplings, longitudinal or transverse fields, etc.) are expected to be independent and identically distributed random variables among which there is no correlation.

There are, however, systems in which the random variables exhibit some kind of correlations, the effect of which can even change the random universality class of the phase transition. Examples of systems with strongly correlated disorder are random quantum magnets at $T=0$, in which the disorder is strictly correlated in the temporal direction. Indeed the critical properties of a such type of random systems are quite unusual, for a review see Ref. 2. In the classical counterpart of these quantum systems, the disorder is strictly correlated in one direction, which leads to models introduced and partially exactly solved by McCoy and $\mathrm{Wu}^{3}$ In a further generalization, the disorder can be strictly correlated in a $d^{\prime}<d$ dimensional subspace, where $d$ is the dimension of the system. 4.5.6.7.8

Correlations between random variables can also be isotropic. One example is presented in Ref. 9 in which the experimental observations are explained ${ }^{10}$ with the assumption of the presence of randomly oriented dislocation lines in the sample. Also in some random quantum magnets ${ }^{11}$ and in the non-Fermi liquid behavior of $f$-electron compounds 12 the disorder is expected to be isotropically correlated due to long-ranged
Ruderman-Kittel-Kasuya-Yoshida (RKKY) interactions. In a coarse-grained picture, the disorder in some coupling strength $\Delta K(\mathbf{r})=K(\mathbf{r})-\overline{K(\mathbf{r})}$ is characterized by its correlator, $G\left(\mathbf{r}-\mathbf{r}^{\prime}\right)=\overline{\Delta K(\mathbf{r}) \Delta K\left(\mathbf{r}^{\prime}\right)}$, which is expected to be isotropic, $G(\mathrm{r})=G(|\mathrm{r}|)$, and to decay algebraically for large arguments: $G(r) \sim r^{-a}$. For example, randomly oriented straight dislocation lines are associated with a correlator exponent $a=d-1$.

The influence of Gaussian disorder with power-law correlations on the critical behavior of the $m$-component vector spin model has been studied in Ref. 13 by fieldtheoretical methods using a double expansion in $\epsilon=4-d$ and $\delta=4-a$. Extending the Harris criterion, correlated disorder with $a \geq d$ is found to be effectively short range and thus relevant only when the pure system has a positive specific heat exponent 1 When $a<d$ and $m=1$ (Ising model) the correlated disorder is shown to be a relevant perturbation at the short-range-disorder fixed point, provided the correlator decays sufficiently slowly, i.e., when

$$
a<\frac{2}{\nu_{\text {short }}},
$$

where $\nu_{\text {short }}$ is the correlation length exponent of the system with short-range disorder. At the stable long-rangedisorder fixed point the correlation length critical exponent is found to be given by

$$
\nu=2 / a, \quad 2 / a>\nu_{\text {short }} .
$$

This result is argued to be exact and thus the extended Harris criterion in Eq. (11) is marginal when the longrange nature of the correlations is relevant. On the other hand the magnetic exponents, such as $x_{m}=\beta / \nu$, depend on both $a$ and $d$.

Recently ${ }_{14}^{14}$ the validity of the scaling relation (2) has been questioned on the basis of a direct renormalization analysis of the scaling functions for the three-dimensional (3D) system in the two-loop approximation, using a Pade-Borel summation technique to evaluate the critical exponents for different values of $a$. 
Numerical and field-theoretical calculations on different types of systems with correlated disorder (percolation, ${ }^{15}$ 3D Ising model, 16 polymers,${ }^{17}$ quantum Ising model, 11 etc.) seem to be in overall agreement with the prediction of Eq. (2).

Here we should make, however, two remarks. First, in the case of the Ising model with randomly distributed dislocation lines, oriented along the lattice axes, 16 for which rotational symmetry is broken, the critical behavior was found to be the same as for the model with Gaussian correlated disorder when $a=2$. This shows that the details of the model of correlated disorder are seemingly irrelevant, the critical behavior being essentially governed by the decay of the disorder correlations. Our second remark concerns the fact that, in systems with correlated disorder, the location of the critical point is generally not known exactly, which introduces a limitation on the accuracy of the numerical calculations.

In the present paper, our aim is to increase the numerical accuracy of the calculation in a statistical mechanical system with correlated disorder and address also such questions (c.f. critical point density profiles, validity of conformal invariance, etc.) which have not been considered previously. For this purpose, we consider the two-dimensional (2D) square lattice Ising model and introduce a type of biaxially correlated disorder which preserves self-duality, thus the critical point is exactly known. Our model can be considered as a 2D self-dual version of the random dislocation model of Ballesteros and Parisi. ${ }^{16}$ In the vicinity of the critical point the properties of the system are explored at the scale of the (diverging) correlation length. A correlator exponent, $a=1$, can be deduced from the integral of the disorder correlator inside a large square of linear size, $L$. Thus at a coarse-grained level our model can be compared to a model with Gaussian correlated disorder for the same value of $a$.

For the 2D Ising model uncorrelated disorder coupled to the local energy density (such as dilution or random ferromagnetic couplings) is a marginally irrelevant perturbation, ${ }^{18,19}$ hence critical singularities of the pure system are supplemented by logarithmic corrections. In particular the correlation length exponent $\nu_{\text {short }}$ keeps its unperturbed value $\nu_{0}=1$. As a consequence from Eq. (11) the biaxially correlated disorder of our model is expected to be relevant. In order to explore the critical properties of the system and to check the validity of Eq. (2) we perform large scale Monte Carlo simulations. In particular the critical exponents are deduced from the finite-size scaling behavior of various magnetic and thermal quantities at the critical temperature, which is exactly known. We also determine the critical profiles of the magnetization and energy densities in order to check whether the forms obtained for the profiles through conformal methods remain valid in the random system after averaging over the disorder.

The structure of the paper is the following. The model, its self-duality, and the relevance-irrelevance criterion are described in Sec.III Finite-size scaling calculations of the critical exponents are presented in Sec.[II] and the critical profiles are studied by conformal methods in Sec.IV. Our results are discussed in Sec. V

\section{MODEL OF SELF-DUAL BIAXIALLY CORRELATED DISORDER}

\section{A. Random-bond Ising models}

We study the spin $1 / 22 D$ Ising model on a square lattice with Hamiltonian

$$
-\beta \mathcal{H}=\sum_{i=1}^{L} \sum_{j=1}^{L}\left(K_{i j}^{x} \sigma_{i, j} \sigma_{i+1, j}+K_{i j}^{y} \sigma_{i, j} \sigma_{i, j+1}\right),
$$

where $\beta=1 / k_{B} T$. The Ising spin $\sigma_{i, j}= \pm 1$ is associated with the site $(i, j)$, located at the intersection between column $i$ and line $j . \quad K_{i j}^{x}$ and $K_{i j}^{y}$ denote the random couplings between $\sigma_{i, j}$ and its first-neighbors $\sigma_{i+1, j}$ in the horizontal direction and $\sigma_{i, j+1}$ in the vertical direction, respectively. In the following we use the parametrization

$$
e^{2 K_{i j}^{\tau}}-1=2^{1 / 2+u_{i j}^{\tau}}
$$

with $\tau=x, y$. Under duality transformation 20 the horizontal and vertical directions are exchanged and we obtain a simple relation between the $u_{i j}^{x}$ and $u_{i j}^{y}$ variables

$$
u_{i j}^{x} \longrightarrow u_{i j}^{y *}=-u_{i j}^{x}, \quad u_{i j}^{y} \longrightarrow u_{i j}^{x *}=-u_{i j}^{y} .
$$

where the superscript, ${ }^{*}$, is used to denote dual variables.

With different distributions of $u_{i j}^{\tau}$ and thus $K_{i j}^{\tau}$ different models are defined, which we list below.

\section{Nonrandom model}

The nonrandom model with $u_{i j}^{x}=u^{x}$ and $u_{i j}^{y}=u^{y}$ has its critical point at $u^{x}=-u^{y}$, which follows from self duality in Eq. (5). For the isotropic model the critical point is at $u^{x}=u^{y}=0$. The critical exponents are $\mathrm{a}^{20}$ $\nu=1$ and $x_{m}=\beta / \nu=1 / 8$.

\section{Random model with uncorrelated disorder}

In this case the $u_{i j}^{\tau}$ parameters are independent and identically distributed (iid) random variables, which are taken from a distribution $P(u)$. At the critical point of the model the distribution is symmetric, ${ }^{21} P(u)=$ $P(-u)$, which is also a consequence of self-duality, see Eq. (5). The strength of disorder $\Delta$ is measured by $\Delta^{2}=\overline{u^{2}}$. In a coarse-grained picture cells, of linear size $\mathrm{L}$, are defined with cell variables, $u^{\prime}=L^{-d} \sum u_{i j}$, where 
the sum runs over $L^{d}$ sites, and $d=2$ is the dimension of the system. In the coarse-grained description we have $\Delta^{\prime}=\Delta L^{-d / 2}$. To decide about relevance-irrelevance of a perturbation according to Harris 1 one should consider the ratio $\Delta^{\prime} / t$ at a length-scale, $L=\xi \sim t^{-\nu}$, where $t$ measures the distance from the critical point. For the 2D Ising model this analysis predicts a marginal effect of the disorder, actually a marginally irrelevant one. As a consequence critical singularities of the nonrandom model are supplemented by logarithmic corrections,$\frac{18,19}{}$

\section{Random model with isotropically correlated disorder}

In this case the $u_{i j}^{\tau}$ parameters are random numbers which are correlated at different sites and we have for large spatial separation

$$
\overline{u_{i j} u_{i^{\prime} j^{\prime}}} \sim\left[\left(i-i^{\prime}\right)^{2}+\left(j-j^{\prime}\right)^{2}\right]^{-a / 2} .
$$

The strength of disorder in the coarse-grained picture

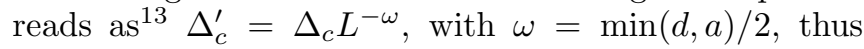
long-range correlation in the disorder can be relevant for $a<d$. Indeed for the 2D Ising model with $a<2$ this type of perturbation is relevant, see Eq. (11) and the correlation length critical exponent is conjectured to be exactly given by Eq. (2). For isotropic correlated disorder the location of the critical point is not known exactly which makes it difficult to analyze numerical results in the critical regime.

\section{McCoy-Wu model}

In the McCoy-Wu mode ${ }^{3}$ the disorder is strictly correlated in columns (or lines)

$$
u_{i j}^{x}=b_{i}^{x}\left(=a_{j}^{x}\right), \quad u_{i j}^{y}=a_{i}^{y}\left(=b_{j}^{y}\right),
$$

where the parameters $b_{i}^{x}, a_{i}^{y}\left(a_{j}^{x}, b_{j}^{y}\right)$ are iid random variables. Now in the coarse-grained description with cells of size $L$, the disorder strength behaves as $\Delta_{M W}^{\prime}=$ $\Delta_{M W} L^{-1 / 2}$. This type of disorder represents a relevant perturbation. According to exact and conjecturedly exact results, this model displays a strongly anisotropic scaling behavior, ${ }^{22}$ so that $\ln \xi_{\perp} \sim \sqrt{\xi}$, where $\xi\left(\xi_{\perp}\right)$ is the correlation length in the nontranslationally invariant (translationally invariant) direction. The critical exponents ${ }^{22}$ are given by: $\nu=2$ and $x_{m}=(3-\sqrt{5}) / 4$.

\section{B. Biaxially correlated disorder} is

Here we introduce a type of correlated disorder which

- Symmetric, with respect to the interchange of the $x$ and $y$ axis,

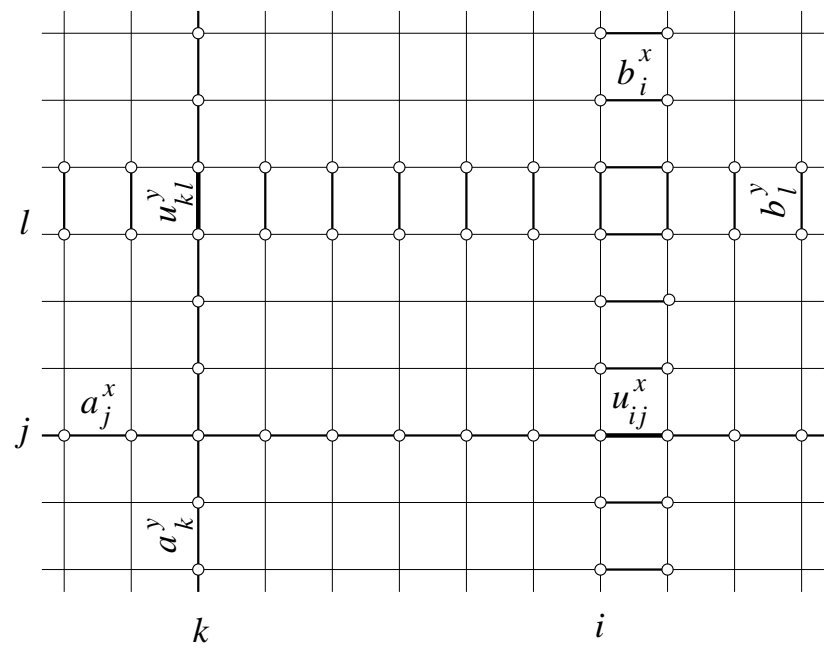

FIG. 1: Parameters entering in the definition of the correlated disorder.

- Has a duality symmetry and

- Represents a 2D version of the random dislocation model ${ }^{16}$

To be concrete we use the parametrization

$$
u_{i j}^{x}=b_{i}^{x}+a_{j}^{x}, \quad u_{i j}^{y}=a_{i}^{y}+b_{j}^{y},
$$

(see Fig. 1), which is the local average (for each bond) of the couplings in two McCoy-Wu models where the disorder is along the $x$ and the $y$ axis, respectively, see Eq. (7). Consequently the strength of disorder is rescaled as in the McCoy-Wu model, $\Delta_{b}^{\prime}=\Delta_{b} L^{-1 / 2}$, but the system has isotropic scaling, as far as the asymptotic behavior of the average correlations is considered. This type of perturbation is relevant (see Sec IC) and has the same scaling exponent as the isotropically correlated random perturbation with the correlation parameter $a=1$ in Eq. (6). We expect that the critical behavior is dictated by the averaged decay of the disorder correlations. As for the 3D random dislocation mode ${ }^{16}$ where the dislocation lines are located along the lattice axes, the breaking of rotational symmetry (before averaging over the quenched disorder) should not change the universality class. The present model, which has the same type of disorder correlations as the Ballesteros-Parisi dislocation model, should also display the same critical behavior as the model with isotropically correlated disorder discussed in Ref. 13 .

An important advantage of the parametrization used in Eq. (8) is that the couplings obey the duality relation in Eq. (5) and thus the system is self-dual for an appropriate choice of the coupling parameters $a_{i}^{y}, a_{j}^{x}, b_{i}^{x}$, and $b_{j}^{y}$. Self-duality can be realized in different ways, leading to a critical system in which both lattice directions are statistically equivalent or not. Here we study the geometry of disorder with the highest symmetry, i.e., with the same symmetric and uniform probability density for all 
the random variables

$$
\begin{aligned}
P(s) & =P(-s)= \begin{cases}\frac{1}{2 \Delta} & -\Delta \leq s \leq \Delta \\
0 & |s|>\Delta\end{cases} \\
s & =a_{i}^{y}, a_{j}^{x}, b_{i}^{x}, b_{j}^{y} .
\end{aligned}
$$

The evolution of the average value $K_{a v}$ and the standard deviation $\Delta K$ of the coupling as a function of the halfwidth $\Delta$ of the probability density is shown in Fig. 2 The standard deviation, with the expansion

$$
\Delta K=.165764 \Delta+.000085 \Delta^{3}+.000010 \Delta^{5}+O\left(\Delta^{7}\right)
$$

is almost linear in $\Delta$. Evidently the relative strength of the disorder $\Delta K / K_{a v}$ is monotonously increasing with $\Delta$.

\section{Relevance-irrelevance criterion}

The stability of the fixed point governing the critical behavior of the pure 2D system in the presence of correlated disorder can be analyzed as by Harris ${ }^{1,13}$ At the length scale $L$, the sum of the deviations from the average coupling grows typically like $\Delta L^{3 / 2}$ since it contains $2 L$ independent random variables with a vanishing average, each variable being of order $\Delta L$ due to the correlations along the $L$ lines and the $L$ columns. Dividing by the number of couplings one defines a typical deviation from the average temperature as

$$
\delta t(L) \simeq \Delta \frac{L^{3 / 2}}{L^{2}} \simeq \Delta L^{-1 / 2} .
$$

The relevance of the perturbation depends on the be-

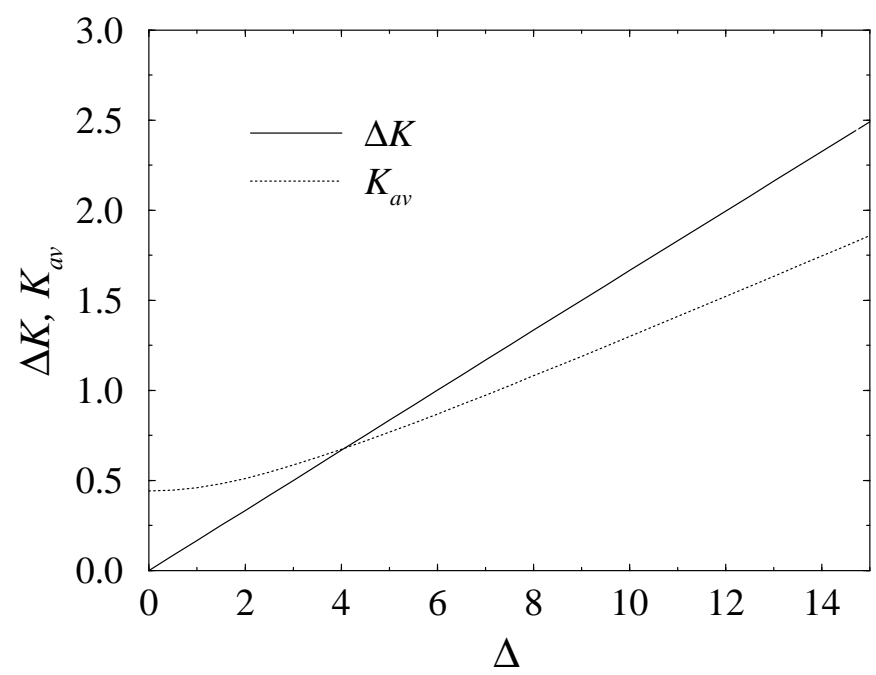

FIG. 2: Variation with $\Delta$ of the average value $K_{a v}$ and the standard deviation $\Delta K$ of the couplings. The standard deviation is almost linear in $\Delta$. havior of the ratio

$$
\frac{\delta t\left(\xi_{0}\right)}{t} \propto t^{-1+\nu_{0} / 2}, \quad \xi_{0} \propto t^{-\nu_{0}},
$$

when one approaches the critical point $(t \rightarrow 0)$. Here $t=\left|T-T_{\mathrm{c}}\right| / T_{\mathrm{c}}$ is the reduced temperature, $\xi_{0}$ the correlation length, and $\nu_{0}$ the correlation length exponent of the unperturbed system. The perturbation is relevant when this ratio diverges at the critical point, i.e., when $\nu_{0}<2$. Thus the biaxial correlated disorder is a relevant perturbation in the case of the $2 \mathrm{D}$ Ising model for which $\nu_{0}=1$.

Alternatively the same conclusion can be drawn from the value of the scaling dimension of $\Delta$ following from Eq. (11). Under a change of the length scale by a factor $b, \delta t$, which is a thermal perturbation, has a scaling dimension $y_{t 0}=1 / \nu_{0}$ and thus transforms as

$$
\delta t^{\prime}=\Delta^{\prime} L^{\prime-1 / 2}=b^{1 / \nu_{0}} \delta t=b^{1 / \nu_{0}} \Delta L^{-1 / 2} .
$$

With $L^{\prime}=L / b$ one obtains

$$
\Delta^{\prime}=b^{y_{\Delta}} \Delta, \quad y_{\Delta}=\frac{2-\nu_{0}}{2 \nu_{0}},
$$

so that, in agreement with the previous result, one finds that the strength of the disorder increases under rescaling when $\nu_{0}<2$.

Here we remind that the biaxial correlated disorder, which is considered in this paper, can be represented by an effective correlator exponent $a=1$ Indeed, the stability limit of the fixed point for Gaussian correlated disorder in Eq. (11) with $a=1$ is the same as the one obtained here for biaxial correlations. As mentioned in the Introduction, this result follows from the comparison of the integral of the disorder correlator at a length scale $L$ for both models. The possible universality of the critical properties of the system with respect to the form of correlated disorder, in particular the validity of the result predicted for the correlation length critical exponent in Eq. (2) will be studied in the following sections.

\section{FINITE-SIZE SCALING AT THE CRITICAL POINT}

\section{A. Monte Carlo technique}

We study the finite-size scaling behavior of different magnetic and thermal quantities at the critical point. We work on a square-shaped system with size $L$ and we use periodic boundary conditions in both directions.

In order to limit the effects of the critical slowing down, the Monte Carlo simulations are performed using the Wolff cluster algorithm. ${ }^{23}$ In order to estimate the equilibration time $\tau_{\mathrm{e}}$ measured in cluster flips units, we compared the evolution of the energy and the magnetization towards equilibrium starting either from a random initial state or from an ordered initial state. We have also 
studied the autocorrelation time for the energy and the magnetization at equilibrium, in order to evaluate the statistical errors on the time averages.

The time averages are calculated during a time $\tau=10^{5}$ to $2.5 \times 10^{5}$ Monte Carlo steps (MCS) after a waiting time of the order of $10 \tau_{\mathrm{e}}\left(10^{3}\right.$ to $\left.5 \times 10^{4} \mathrm{MCS}\right)$. The disorder averages are taken over $n_{\mathrm{s}} \approx 10^{4}$ samples. These values were chosen in order to obtain a precision close to $1 \%$ for the different quantities studied.

The simulations are performed in a vanishing external field and we use the fluctuation-dissipation relations to evaluate the specific heat and the magnetic susceptibility. The averaged value of a quantity $X$ is the result of a time average for a sample $s$ followed by an average over $n_{\mathrm{s}}$ realizations of the disorder so that:

$$
\overline{\langle X\rangle}=\frac{1}{n_{\mathrm{s}}} \sum_{s=1}^{n_{\mathrm{s}}}\left\langle X^{(s)}\right\rangle, \quad\left\langle X^{(s)}\right\rangle=\frac{1}{\tau} \sum_{i=1}^{\tau} X_{i}^{(s)} .
$$

The total magnetization $M$ and the total energy $E$ are defined as

$$
M=\sum_{i, j=1}^{L} \sigma_{i, j}, \quad E=-\sum_{i, j=1}^{L}\left(\sigma_{i, j} \sigma_{i+1, j}+\sigma_{i, j} \sigma_{i, j+1}\right) .
$$

The quantities studied are the following:

- The moments of the magnetization per site

$$
m_{p}=\frac{\overline{\left\langle|M|^{p}\right\rangle}}{L^{2 p}}, \quad m_{1}=m .
$$

- The susceptibility per site

$$
\chi=\frac{\overline{\left\langle\Delta M^{2}\right\rangle}}{L^{2}}, \quad \Delta M^{2}=(M-\langle M\rangle)^{2} .
$$

- The specific heat per site

$$
C=\frac{\overline{\left\langle\Delta E^{2}\right\rangle}}{L^{2}}, \quad \Delta E^{2}=(E-\langle E\rangle)^{2} .
$$

- The temperature derivative of the logarithm of the susceptibility:

$$
\Psi=\frac{\overline{\left\langle\Delta E \Delta M^{2}\right\rangle}}{\overline{\left\langle\Delta M^{2}\right\rangle}}, \quad \Delta E=E-\langle E\rangle .
$$

\section{B. Simulations results}

In Fig. 3equilibrium spin configurations for the system with biaxially correlated disorder at $\Delta=9$ are compared to the corresponding pure system configurations in the low-temperature phase, at the critical point and in the high-temperature phase for a system with size $116 \times 116$ with periodic boundary conditions. In the off-critical systems, spin clusters are larger for the disordered system
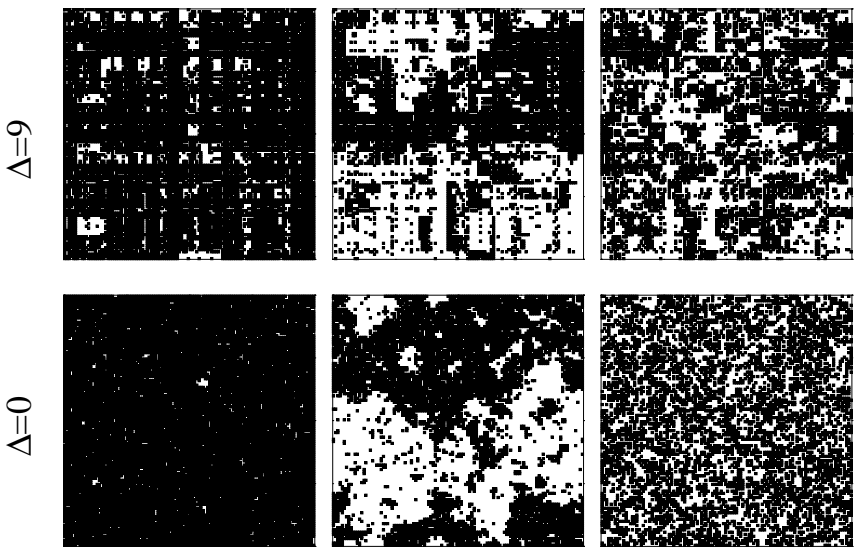

$T / T_{c}=0.8$

$T / T_{c}=1$

$T / T_{c}=2$

FIG. 3: Snapshots of equilibrium spin configurations for the random system $(\Delta=9)$ and the pure system $(\Delta=0)$ in the ordered phase $\left(T=0.8 T_{\mathrm{c}}\right)$, at the critical point and in the paramagnetic phase $\left(T=2 T_{\mathrm{c}}\right)$.

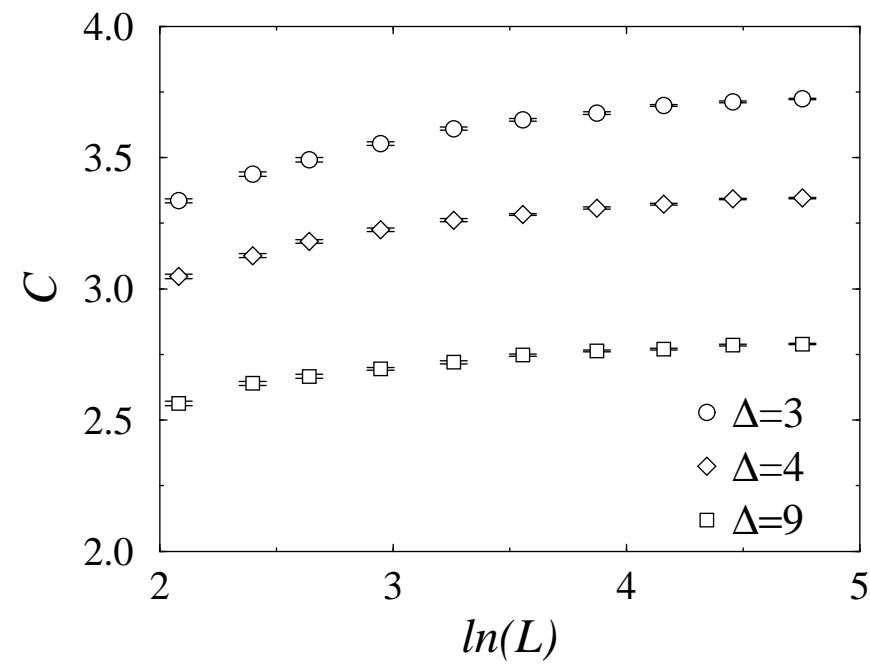

FIG. 4: Semilogarithmic plot of the finite-size behavior of the specific heat per spin at the critical point. The specific heat exponent $\alpha$ is negative and the behavior of $C$ is dominated by its regular contribution for large values of $L$.

whereas the long-range aspect is roughly the same for both systems at the critical temperature.

The finite-size behaviors at the critical point of the specific heat $C$, the moments of the magnetization density $m_{p}$, the susceptibility $\chi$, and the temperature derivative of $\ln \chi, \Psi$, are shown in Figs. 47 for three values of the disorder amplitude, $\Delta=3,4$ and 9 , and for ten sizes ranging from $L=8$ to $L=116$.

The specific heat saturates at large system size, which indicates that the regular contribution dominates the singular one. The singular contribution, behaving as $L^{\alpha / \nu}$, vanishes at large system size, i.e., the specific heat exponent $\alpha$ is negative. This is the reason why we studied 


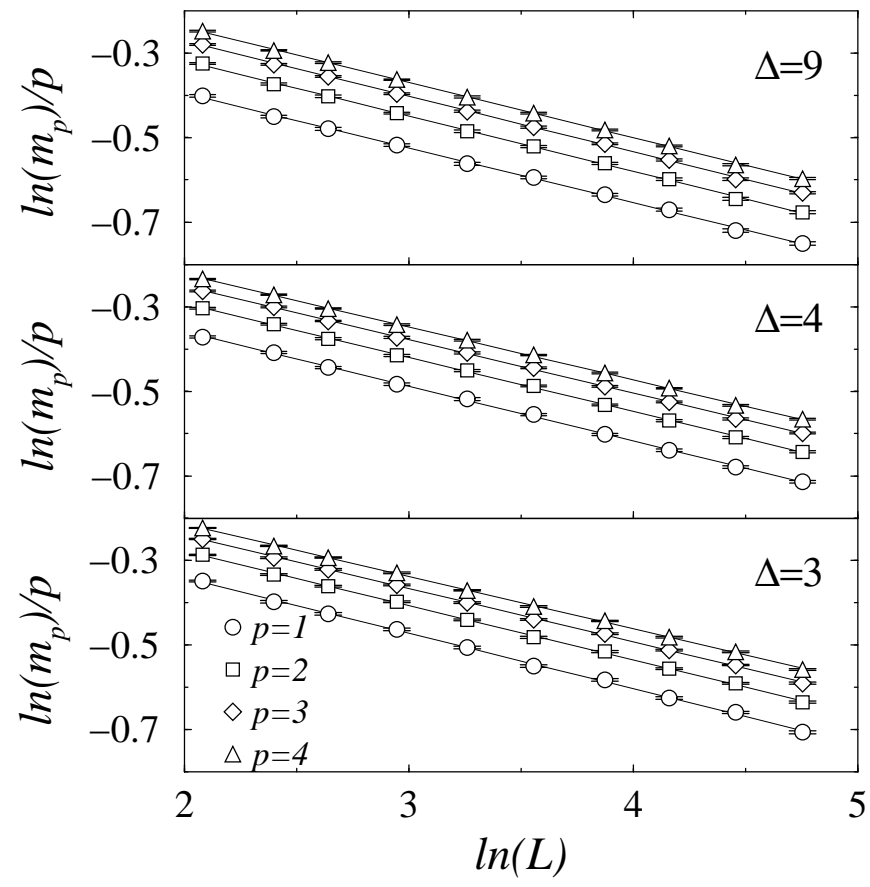

FIG. 5: Finite-size scaling of the moments of the magnetization density at the critical point. The lines correspond to linear fits of the data.

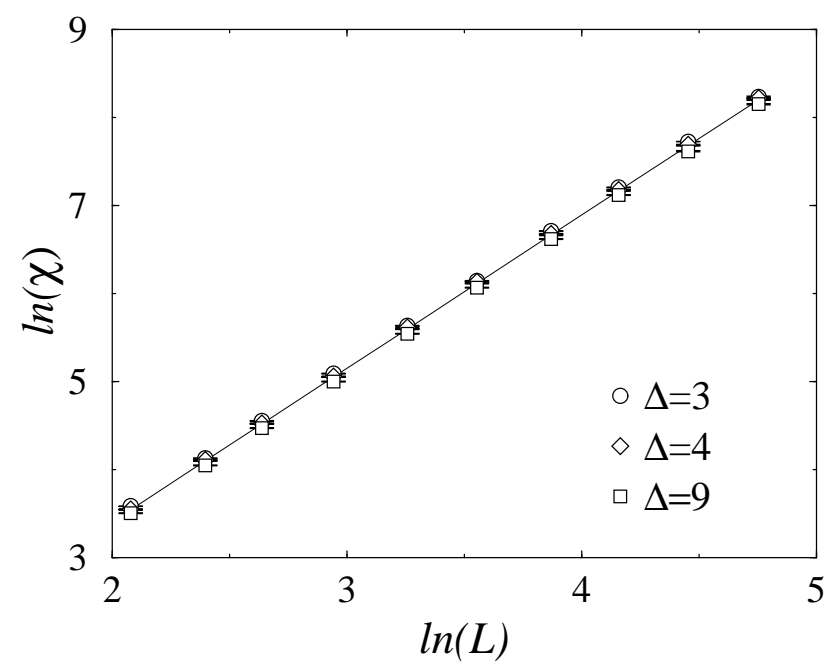

FIG. 6: Finite-size scaling of the magnetic susceptibility per spin at the critical point. The influence of $\Delta$ is quite small and the line gives the linear fit of all the data.

the finite-size behavior of $\Psi$ which diverges with the size of the system, thus allowing us to estimate the thermal exponent $1 / \nu$.

According to finite-size scaling theory, the following behaviors are expected for a large critical system with

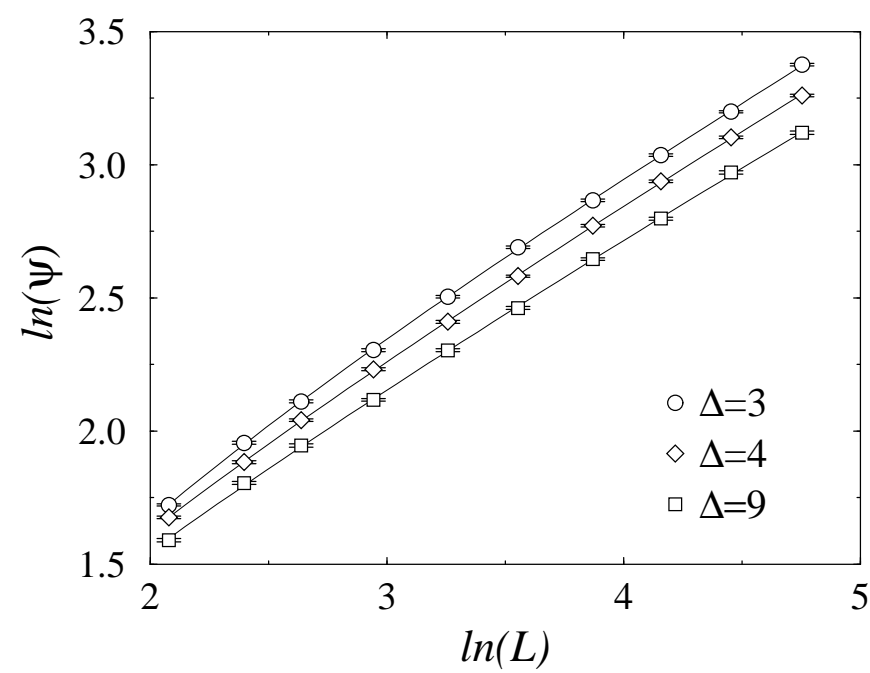

FIG. 7: Finite-size scaling of $\Psi$, temperature derivative of the magnetic susceptibility at the critical point. The lines are the nonlinear fits of the data as explained in the text.

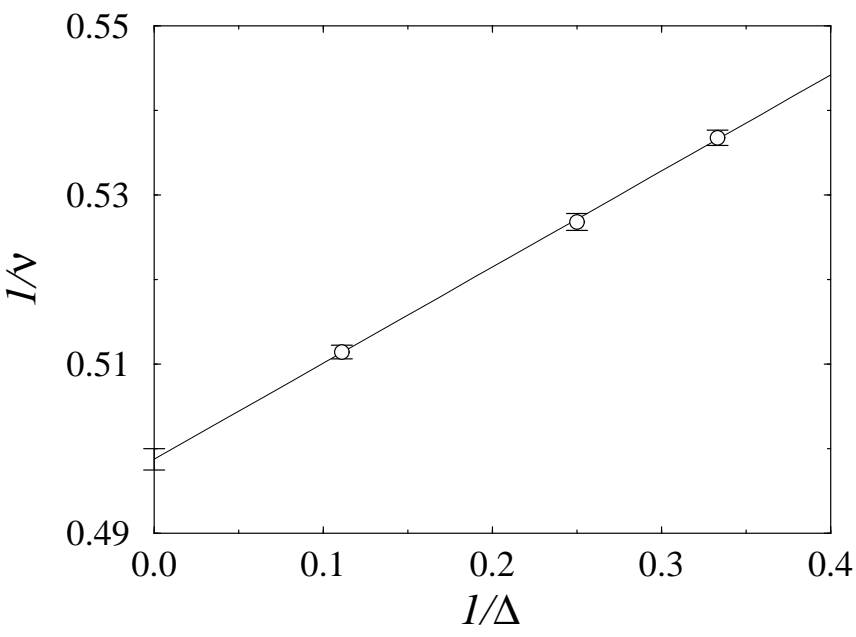

FIG. 8: Linear extrapolation of $1 / \nu$ to infinite disorder strength.

size $L$ :

$$
\begin{array}{rlrl}
m_{p} & \propto L^{-p x_{m}^{(p)}}, & & x_{m}^{(1)}=x_{m}=\beta / \nu \\
\chi \propto L^{\gamma / \nu}, & & \gamma / \nu=d-2 x_{m}=2\left(1-x_{m}\right), \\
\Psi \propto L^{1 / \nu}, & & 1 / \nu=y_{t} .
\end{array}
$$

The log-log plots for $\left(m_{p}\right)^{1 / p}, \chi$, and $\Psi$ display the expected linear behavior, although with noticeable deviations at small size and a slightly $\Delta$-dependent slope for $\Psi$. Thus the magnetization and susceptibility exponents were simply obtained through linear fits, whereas we used a nonlinear fit of the data for $\Psi$

$$
\ln (\Psi)=A+\frac{1}{\nu} \ln (L)+\ln \left(1+B L^{-\omega}\right) .
$$


TABLE I: Effective critical exponents deduced from the fits of the finite-size scaling data in Figs. 5] The last line gives the values of the effective correction-to-scaling exponent for $\Psi$ defined in Eq. (22).

\begin{tabular}{llll}
\hline \hline & $\Delta=3$ & $\Delta=4$ & $\Delta=9$ \\
\hline$\beta / \nu$ & $0.1312(13)$ & $0.1292(10)$ & $0.1294(14)$ \\
$x_{m}^{(2)}$ & $0.1283(10)$ & $0.1279(8)$ & $0.1309(11)$ \\
$x_{m}^{(3)}$ & $0.1259(8)$ & $0.1264(8)$ & $0.1310(9)$ \\
$x_{m}^{(4)}$ & $0.1238(7)$ & $0.1249(7)$ & $0.1305(8)$ \\
$\gamma / \nu$ & $1.7435(21)$ & $1.7442(17)$ & $1.7381(22)$ \\
$1 / \nu$ & $0.5368(9)$ & $0.5268(10)$ & $0.5115(8)$ \\
$\omega$ & $0.737(7)$ & $0.573(6)$ & $0.569(7)$ \\
\hline \hline
\end{tabular}

The exponents are collected in Table@for the three values of the disorder strength. There is no significant variation of $\beta / \nu$ and $\gamma / \nu$ with $\Delta$. Taking the average of the exponents obtained for the three values of $\Delta$ leads to

$$
\frac{\beta}{\nu}=x_{m}=0.1299(12), \quad \frac{\gamma}{\nu}=1.7419(20) \text {. }
$$

There is no clear evidence of multiscaling: The slow decay of $x_{m}^{(p)}$ with increasing $p$ is probably due to a crossover effect since it disappears at $\Delta=9$ when the disorder is sufficiently strong.

The variation of $1 / \nu$ with $\Delta$ is significant. As shown in Fig. 8 it is linear in $1 / \Delta$ and the extrapolation leads to

$$
\frac{1}{\nu}=y_{t}=0.4988(13),
$$

at infinite disorder strength.

\section{CRITICAL PROFILES}

The analysis of Monte Carlo data for the critical profiles using the tools of conformal theory provides an efficient method for the determination of bulk and surface critical exponents 24.25 .26

Using the replica trick for a system with biaxially correlated disorder leads to an effective Hamiltonian with long-range interactions between the replicas. Thus we do not expect our system to be conformally invariant at its new fixed point as it seems to be the case for uncorrelated disorder ${ }^{24}$ Nonetheless, in this section we assume that the critical profiles have a leading behavior which is the same as for a conformally invariant system. In both cases the form of the profiles is constrained by covariance under global scale transformations and by the values of surface and bulk exponents. We know of at least one example, the random Ising chain in a transverse field, for which the critical profiles follow quite accurately the conformal predictions, 27 although the system cannot be conformally invariant since it displays a strongly anisotropic scaling behavior ${ }^{22,28}$
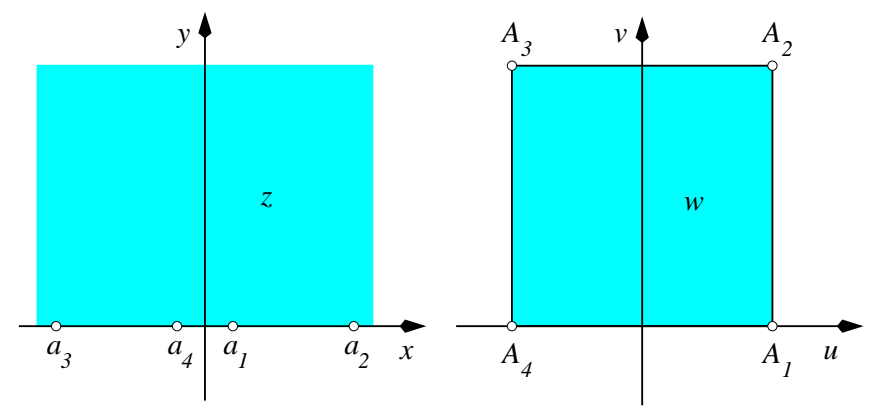

FIG. 9: Conformal transformation of the half-plane into a square. The boundary conditions, free or fixed, are the same on both systems.

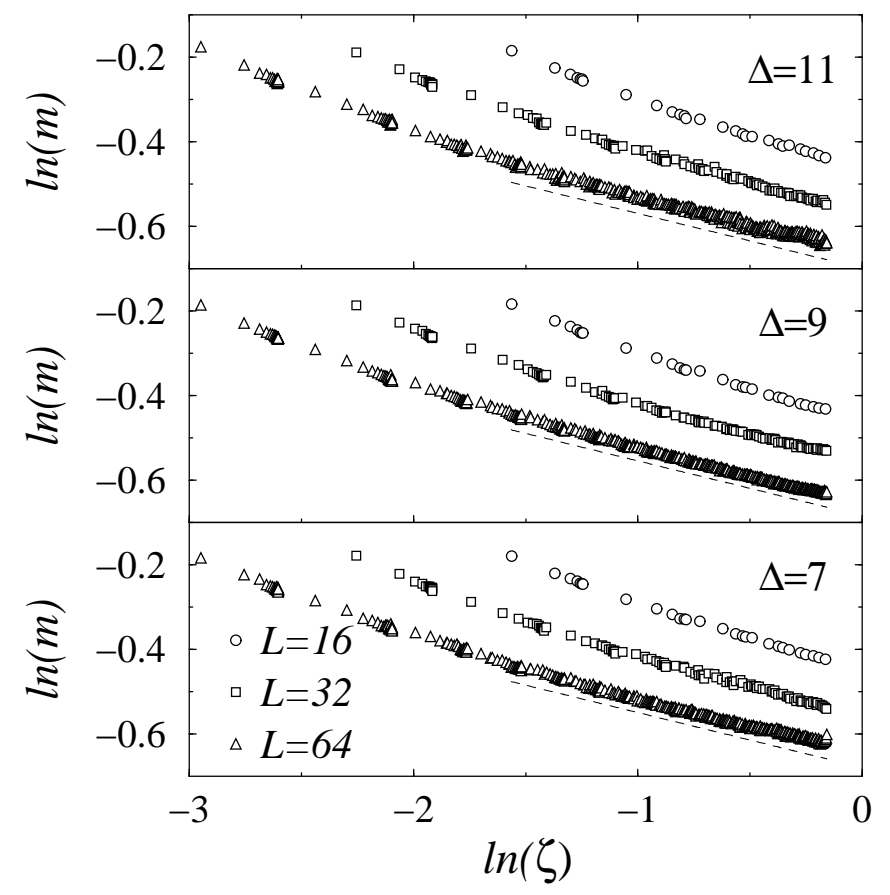

FIG. 10: Conformal profiles of the magnetization density for different system sizes $L$ and disorder strength $\Delta$. The dashed lines indicate the slope $-\beta / \nu$ expected from the finite-size scaling results.

\section{A. Conformal transformation of the densities}

On a semi-infinite critical system with fixed boundary conditions, the form of the magnetization profile is fixed, up to a constant amplitude, by translational, rotational, and global scale invariance. It decays as a power of the distance $y$ from the surface ${ }^{29}$

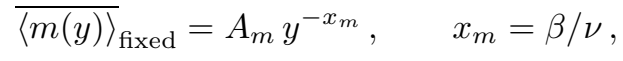

where the exponent $x_{m}$ is the scaling dimension of the bulk magnetization density.

Let us consider the Schwarz-Christoffel transformation 


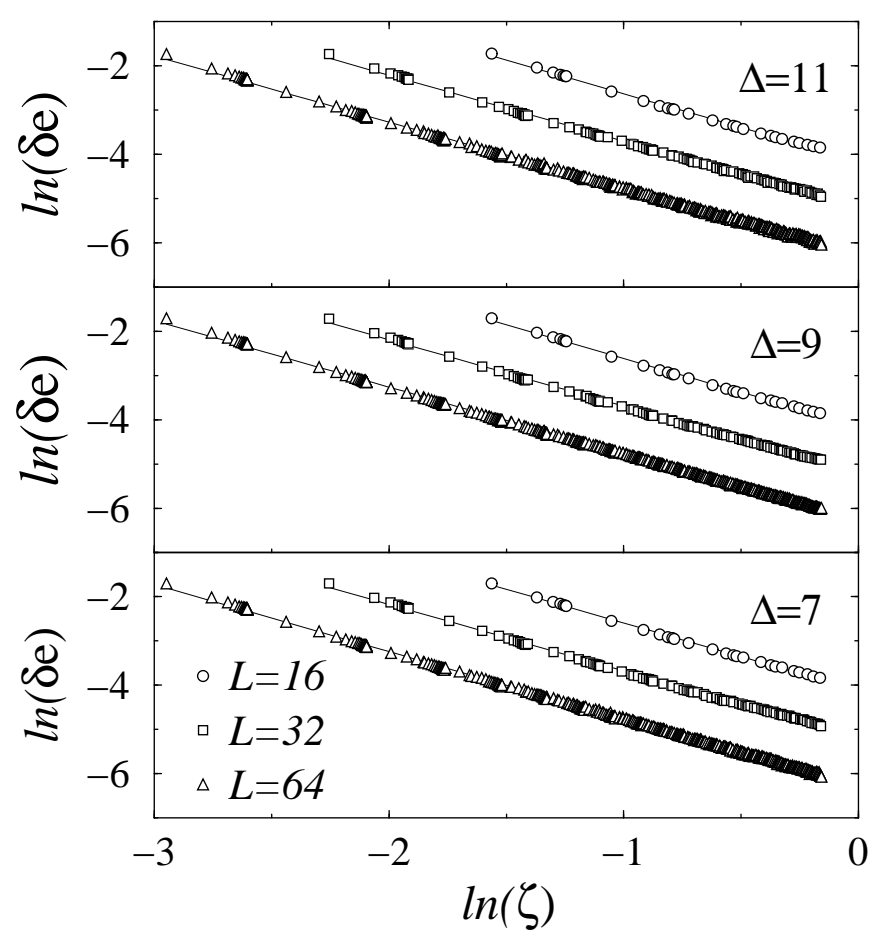

FIG. 11: Conformal profiles of the singular part of the energy density for different system sizes $L$ and disorder strength $\Delta$. The lines correspond to the linear fits of the data.

of the half-plane $z=x+i y$ such that

$$
w(z)=\frac{L}{2 K(k)} F(z, k), \quad z=\operatorname{sn}\left(\frac{2 K w}{L}\right),
$$

where $w(z)=u+i v$ and

$$
F(z, k)=\int_{0}^{z} \frac{d t}{\sqrt{\left(1-t^{2}\right)\left(1-k^{2} t^{2}\right)}}
$$

is the elliptic integral of the first kind with modulus $k$. $K(k)=F(1, k)$ is the complete elliptic integral of the first kind and sn is the Jacobian elliptic sine.

This conformal transformation maps the half-plane $y=\Im(z) \geq 0$ onto the interior of the square with $-L / 2 \leq u \leq L / 2,0 \leq v \leq L$ (see Fig. 9) when the modulus $k$ verifies the relation $K(k) / K\left(\sqrt{1-k^{2}}\right)=1 / 2$ (see e.g. Ref. 30). by

In the new geometry the magnetization density is given

$$
\begin{aligned}
{\overline{\langle m(w)\rangle_{\text {fixed }}}} & =b(z)^{x_{m}}{\overline{\langle m(z)\rangle_{\text {fixed }}}}, \quad b(z)=\left|w^{\prime}(z)\right|^{-1} \\
w^{\prime}(z) & =\frac{L}{2 K(k) \sqrt{\left(1-z^{2}\right)\left(1-k^{2} z^{2}\right)}}
\end{aligned}
$$

Finally, introducing the reduced variable

$$
\zeta(w)=\frac{\Im[z(w)]}{\left|\sqrt{\left(1-z^{2}\right)\left(1-k^{2} z^{2}\right)}\right|},
$$

TABLE II: Scaling dimension of the energy density $x_{e}$ deduced from the linear fits of the conformal profiles in Fig. 11

\begin{tabular}{llll}
\hline \hline$L$ & $\Delta=7$ & $\Delta=9$ & $\Delta=11$ \\
\hline 16 & $1.5435(9)$ & $1.5377(9)$ & $1.5177(10)$ \\
32 & $1.5241(9)$ & $1.5207(6)$ & $1.5110(9)$ \\
64 & $1.5209(6)$ & $1.5182(4)$ & $1.5002(8)$ \\
\hline \hline
\end{tabular}

the magnetization density varies as a power of $\zeta$

$$
m={\overline{\langle m(\zeta)\rangle_{\text {fixed }}}} \propto \zeta^{-x_{m}} .
$$

The same behavior is obtained for the singular part of the energy density, for which

$$
{\overline{\langle e(\zeta)\rangle_{\operatorname{sing}}}} \propto \zeta^{-x_{e}}, \quad x_{e}=2-1 / \nu .
$$

In this case one needs to eliminate the regular contribution to the energy density. The regular part can be canceled by taking the difference $\delta e$ between the energy density profiles obtained on a system with either free boundary conditions $e_{\text {free }}(w)$ or fixed boundary conditions $e_{\text {fixed }}(w)$ and then

$$
\delta e=\overline{\left\langle e_{\text {free }}(\zeta)\right\rangle}-\overline{\left\langle e_{\text {fixed }}(\zeta)\right\rangle} \propto \zeta^{-x_{e}} .
$$

The amplitudes of the singular parts have opposite signs with the two types of boundary conditions ${ }^{26.31}$ thus in the difference the singular contribution is amplified and the bulk regular contribution is eliminated.

\section{B. Monte Carlo simulations}

We work on a square lattice with either fixed or free boundary conditions using the Swendsen-Wang cluster algorithm ${ }^{32}$ which is more efficient than the Wolff algorithm with fixed boundary conditions. The simulations were performed for three lattice sizes $(L=16,32,64)$ and three values of the couplings $(\Delta=7,9,11)$. After a waiting time $\tau_{\mathrm{e}}=5 \times 10^{3} \mathrm{MCS}$, the time averages are taken during a time $\tau=6 \times 10^{4}$ MCS. The disorder averages are taken over a number of samples ranging from $n_{\mathrm{s}}=2 \times 10^{4}$ for the smaller systems to $n_{\mathrm{s}}=2 \times 10^{3}$ for the larger ones.

For the magnetization density profiles all the surface spins are fixed in the +1 state. The behavior of the singular part of the energy density is obtained by taking the difference of Eq. (32) between two systems with either free or fixed boundary conditions for the same realizations of the disorder, which considerably reduces the statistical noise.

The log-log plots for the magnetization and the energy densities are shown in Figs. 1011 as functions of the reduced conformal variable $\zeta$ defined in Eq. (29).

The magnetization density profiles show strong corrections to scaling and due to the small values of $\zeta$ we were 
TABLE III: Critical exponents deduced from the scaling laws using the finite-size scaling results for $\beta / \nu, \gamma / \nu$ in Eq. 23) and $1 / \nu$ in Eq. (24) as inputs.

\begin{tabular}{llllllll}
\hline \hline$x_{m}$ & $x_{e}$ & $\alpha$ & $\beta$ & $\gamma$ & $\delta$ & $\nu$ & $\eta$ \\
\hline $0.1294(7)$ & $1.5012(13)$ & $-2.01(1)$ & $0.2604(31)$ & $3.492(13)$ & $14.46(8)$ & $2.005(5)$ & $0.2588(14)$ \\
\hline \hline
\end{tabular}

unable to find a reliable nonlinear fit of our data. Nevertheless, the dashed lines indicate that the asymptotic slopes are in agreement with the value of $\beta / \nu$ obtained by finite-size scaling and given in Eq. (23).

On the contrary, the corrections are very weak for the energy density profiles in Fig. 11 so that the slopes could be obtained with a good accuracy through a linear fit of the data. The small deviations from linearity occur close to the surface for small values of $\zeta$. Only a few points are concerned and their weight in the fit is negligible. The values of $x_{e}$ are collected in Table Ifor the different values of $L$ and $\Delta$. They are slowly decreasing when $\Delta$ or $L$ increases.

\section{DISCUSSION}

Our results are collected in Table III The scaling dimension of the magnetization density $x_{m}$ is directly given by $\beta / \nu$ in Eq. (23). Alternatively, it can estimated using $\gamma / \nu$ together with the scaling law $\gamma / \nu=d-2 x_{m}$, which gives $x_{m}=0.1291(10)$. The value in Table II corresponds to the centre of the confidence interval which is common to the two estimates. It is quite close to the value for the pure $2 \mathrm{D}$ Ising model $x_{m}=1 / 8$. Thus the magnetization in the random and pure systems have almost the same fractal dimensions. This explains why both systems in Fig. 3 have roughly the same aspect at the critical point. The value of $x_{m}$ was used to calculate $\eta=2 x_{m}+2-d=2 x_{m}$ and $\delta=-1+d / x_{m}$.

The value of $\nu$ following from Eq. (24) is such that, according to Eq. (14), the disorder is irrelevant at the new fixed point, as required for its stability. Furthermore the numerical estimate of $\nu$, within the error of the calculation corresponds to the prediction in Eq. (2) with the effective correlator exponent, $a=1$. The estimate of $\nu$ leads to the value of the specific heat exponent through the Josephson scaling law $\alpha=2-d \nu$. The correlation length diverges more strongly in the random system with $\nu$ close to 2 than in the pure system with $\nu=1$, this might explain the difference in the cluster sizes for the off-critical systems in Fig. 3

The scaling dimension of the energy density $x_{e}$ is ob- tained using the scaling law $x_{e}=d-1 / \nu$. The values of $x_{e}$ deduced from the conformal profiles, collected in Table III are close to this estimate for large sizes and strong enough disorder.

To summarize we have studied the critical behavior of the square lattice Ising model with biaxially correlated disorder by large scale MC simulations. The different estimates of the critical exponents give a consistent picture, in which $\nu$ seems to be the same as for isotropic Gaussian correlated disorder, whereas $x_{m}$ is not very far from the pure and uncorrelated random systems values.

One important technical simplification of the model is its self-duality property, which is used to locate exactly the critical point. The same type of parametrization, as given in Eq. (8) can be used to preserve self-duality in the $q$-state Potts model, for any value of $q^{33}$ An investigation of these models could provide further information about the nature of the phase transition in disordered systems. For example, $q=1$ is equivalent to the percolation problem, 34 whereas the phase transition in the pure system is of first order for $q>4$ In the latter problem, due to uncorrelated disorder, the transition is softened into a second-order one, $\stackrel{36}{r}$ with a correlation length exponent $\nu_{\text {short }} \approx 1.37$ Consequently, according to the extended Harris criterion in Eq. (1), the phase transition is expected to belong to a new and $q$-dependent universality class. If the limiting behavior for large $q$ can be predicted, as for uncorrelated disorder ${ }^{38}$ remains the subject of a separate study.

\section{Acknowledgments}

We thank Christophe Chatelain for useful discussions and Bertrand Berche for giving us his tabulated values of $\zeta$. F.Á.B. thanks the Ministère Français des Affaires Etrangères for a research grant. This work has been supported by the Hungarian National Research Fund under Grant Nos. OTKA TO34183, TO37323, TO48721, MO45596, and M36803. Intensive simulations have been performed at CINES Montpellier under Project No. pnm2318. The Laboratoire de Physique des Matériaux is Unité Mixte de Recherche CNRS No. 7556.

\footnotetext{
* Electronic address: turban@lpm.u-nancy.fr

† Electronic address: igloi@szfki.hu

1 A. B. Harris, J. Phys. C 7, 1671 (1974).
}

${ }^{2}$ F. Iglói, and C. Monthus, Phys. Rep. 412, 277 (2005).

3 B. M. McCoy and T. T. Wu, Phys. Rev. 176, 631 (1968); 188, 982 (1969); B. M. McCoy, ibid. 188, 1014 (1969). 
4 S. N. Dorogovtsev, Fiz. Tverd. Tela (Leningrad) 22, 321 (1980) [Sov. Phys. Solid State 22, 188 (1980)]; Phys. Lett. 76A, 169 (1980).

${ }^{5}$ D. Boyanovsky and J. L. Cardy, Phys. Rev. B 26, 154 (1982).

6 J. C. Lee and R. L. Gibbs, Phys. Rev. B 45, 2217 (1992).

7 V. Blavats'ka, C. von Ferber, and Yu. Holovatch, Phys. Rev. B 67, 094404 (2003).

8 R. Sknepnek and T. Vojta, Phys. Rev. B 69, 174410 (2004).

9 T. R. Thurston, G. Helgesen, J. P. Hill, D. Gibbs, B. D. Gaulin, and P. J. Simpson, Phys. Rev. B 49, 15730 (1994); K. Hirota, G. Shirane, P. M. Gehring, and C. F. Majkrzak, ibid. 49, 11967 (1994).

10 M. Altarelli, M. D. Núñez-Regueiro, and M. Papoular, Phys. Rev. Lett. 74, 3840 (1995).

11 H. Rieger and F. Iglói, Phys. Rev. Lett. 83, 3741 (1999).

12 A. H. Castro Neto, G. Castilla, and B. A. Jones, Phys. Rev. Lett. 81, 3531 (1998); M. C. de Andrade, R. Chau, R. P. Dickey, N. R. Dilley, E. J. Freeman, D. A. Gajewski, M. B. Maple, R. Movshovich, A. H. Castro Neto, G. Castilla, and B. A. Jones, ibid. 81, 5620 (1998).

13 A. Weinrib and B. I. Halperin, Phys. Rev. B 27, 413 (1983).

14 V. V. Prudnikov, P. V. Prudnikov, and A. A. Fedorenko, Phys. Rev. B 62, 8777 (2000).

15 A. Weinrib, Phys. Rev. B 29, 387 (1984).

16 H. G. Ballesteros and G. Parisi, Phys. Rev. B 60, 12912 (1999).

17 V. Blavats'ka, C. von Ferber, and Yu. Holovatch Phys. Rev. E 64, 041102 (2001).

18 Vik. S. Dotsenko and Vl. S. Dotsenko, Adv. Phys. 32, 129 (1983); V. Dotsenko, Usp. Fiz. Nauk 165, 481 (1995); B. N. Shalaev, Phys. Rep. 237, 129 (1994).
19 W. Selke, L. N. Shchur, and A. L. Talapov, in Annual Reviews of Computational Physics, Vol. 1, edited by D. Stauffer (World Scientific, Singapore, 1994), p. 17.

20 See R.J. Baxter, Exactly Solved Models in Statistical Mechanics, (Academic Press, London, 1982).

21 W. Kinzel and E. Domany, Phys. Rev. B 23, 3421 (1981).

22 D. S. Fisher, Phys. Rev. Lett. 69, 534 (1992); Phys. Rev. B 51, 6411 (1995).

23 U. Wolff, Phys. Rev. Lett. 60, 1461 (1988).

24 C. Chatelain and B. Berche, Phys. Rev. E 60, 3853 (1999).

25 I. Reš and J. P. Straley, Phys. Rev. B 61, 14425 (2000).

26 B. Berche, J. Phys. A 36, 585 (2003).

27 F. Iglói and H. Rieger, Phys. Rev. B 57, 11404 (1998).

28 A. P. Young and H. Rieger, Phys. Rev. B 53, 8486 (1996).

29 M. E. Fisher and P. G. de Gennes, C. R. Acad. Sci. Paris B 287, 207 (1978).

30 B. A. Fuchs and B. V. Shabat, Functions of a Complex Variable (Pergamon, Oxford, 1964).

31 D. Karevski, P. Lajkó, and L. Turban, J. Stat. Phys. 86, 1153 (1996).

32 R. H. Swendsen and J.-S.Wang, Phys. Rev. Lett. 58, 86 (1987).

33 F. Y. Wu, Rev. Mod. Phys. 54, 235 (1982).

34 P. W. Kasteleyn and C. M. Fortuin, J. Phys. Soc. Jpn. 46 (suppl.), 11 (1969).

35 R.J. Baxter, J. Phys. C 6, L445 (1973).

36 M. Aizenman and J. Wehr, Phys. Rev. Lett. 62, 2503 (1989); 64, 1311(E) (1990).

37 For a review, see: J. L. Cardy, Physica A 263, 215 (1999).

38 J.-Ch. Anglès d'Auriac and F. Iglói, Phys. Rev. Lett. 90, 190601 (2003); M.-T. Mercaldo, J.-Ch. Anglés d'Auriac, and F. Iglói, Phys. Rev. E 69, 056112 (2004). 